\title{
Rayleigh criterion of resolution and light sources of different spectral composition
}

Pablo Meilan, Mario Garavaglia

Pablo F. Meilan, Mario Garavaglia, "Rayleigh criterion of resolution and light sources of different spectral composition," Proc. SPIE 3190, Fifth International Topical Meeting on Education and Training in Optics, (8 December 1997); doi: $10.1117 / 12.294397$

Event: Fifth International Topical Meeting on Education and Training in Optics, 1997, Delft, Netherlands 
The Rayleigh Criterion of Resolution and Light Sources of Different Spectral Composition

\author{
Pablo F. Meilán and Mario Garavaglia \\ Departmento de Física, Facultad de Ciencias Exactas \\ Universidad Nacional de La Plata \\ CC 67, Correo Central, 1900 La Plata, Argentina \\ and \\ Centro de Investigaciones Opticas (CIOp) \\ CC 124, Correo Central, 1900 La Plata, Argentina \\ Tel/Fax: + (54) (21) 714341 \\ E-mail: laserlab@pinos.com
}

\begin{abstract}
The results of very simple experiments to evaluate Lord Rayleigh Resolution Criterion validity are discussed in cases of quasimonochromatic sources of small angular dimensions (LEDs) and monochromatic sources (Lasers), the emissions of which have different or equal spectral compositions. Visual observations as well as color photographs and color video recording were utilized in the experiments. The validity of the resolution criterion was confirmed when the diffraction patterns were produced using LEDs and lasers of the same color (red or green). However, when LEDs and lasers of different color were used, better resolutions than those of Rayleigh Criterion were obtained owing to the non-spectral yellow false color resulting from the overlapping of the red and green spectral colors. Therefore, the observation of the non-spectral false color implies the super-resolution process.
\end{abstract}

Keywords: Rayleigh criterion, Super-resolution.

\title{
1. INTRODUCTION
}

In 1879, John William Strutt (Third Baron Rayleigh) investigated the limit of resolution (resolving or separating power) of some optical instruments, such as telescopes and spectroscopes. ${ }^{1}$ The telescope pupil being circular, the image of a star appears as a bright disk surrounded by lesser intensity rings. This irradiance distribution corresponds to the diffraction pattern of a circular pupil or Airy distribution:

$$
I(\theta)=I(0)\left[2 J_{1}(k \cdot a \cdot \sin \theta) / k \cdot a \cdot \sin \theta\right]^{2},
$$

in which $k=2 \pi / \lambda$, where $\lambda$ is the light wavelength and $a$ the pupil radius. In such case, the angular radius $\theta$ of the bright disk, is equal to:

$$
\theta=1.2197 \lambda / 2 a \approx 1.22 \lambda / 2 a .
$$

Lord Rayleigh ${ }^{1}$ observed that the images produced by two stars emitting in the same spectral region will have their diffraction bright disks in contact when the stars angular separation is equal to $2 \theta$, in which case there will be no doubt that they are resolved (unless their magnitudes are notably different). This diffraction central disks condition of contact is known as the null first derivative, in so far as in such situation the Airy distribution first zero of one of the images coincides with the central peak of the other. In spite of this interesting result, Lord Rayleigh commented that "this rule is convenient on account of its simplicity; and it is sufficiently accurate in view of the necessary uncertainty as to what exactly is meant by resolution". And he added that "perhaps in practice somewhat more favourable conditions are necessary to secure a resolution that would be thought satisfactory". In this respect, it can be affirmed that the Sparrow resolution criterion represents an improvement. The Sparrow criterion is based on the null second derivative condition to establish that both images of two point light sources affected by diffraction are resolved. 
Lord Rayleigh ${ }^{1}$ discussed the concept of the spacial resolution of optical sources from the point of view of the diffraction produced at the entrance aperture of the instruments. In case of eyes, cameras, telescopes and microscopes apertures are circular; while in case of different types of spectral analysis instruments, they are rectangular due to their slits, prisms or diffrantion gratings. Lord Rayleigh stated in every case, "If the circular beam be received upon a perfect lens, an image is formed in the focal plane in wich directions are represented by points. The image accordingly consists of a central disk of light surrounded by luminous rings of rapidly diminishing brightness. It was under this form that the problem was originally investigated by Airy." and "The diffraction phenomena presented by beams of rectangular section are simpler in theory that when the section is circular; and they have a practical application in the spectroscope, when the beam is limited by prisms or gratings rather than by the object-glasses of the telescopes". After his calculations of the irradiance distribution according to the $u^{-2} \sin ^{2} u$ function for a rectangular aperture, Lord Rayleigh wrote: "The brightness midway between the two central points is 0.8106 of the brightness at the central points themselves". This is a perfectly correct sentence. However, it was misunderstanded along the years. Authors beleve that perhaps it ocurred due to the repetition of the words central points. Obviously the two central points in the description of Lord Rayleigh correspond to the peaks at $u=0$ and $u=\pi / 4$ in the irradiance distribution curves. Nevertheless, in very splendid books on Optics this description was interpreted as to be applied to point light sources observed through circular apertures, as in the Optique by Bruhat $^{2}$; while in other books such description is accepted as to be valid for circular and rectangular apertures, as in the Light by Ditchburn ${ }^{3}$. Born and Wolf ${ }^{4}$ in a short footnote save the historical error. In fact, they stated that for a slit aperture the saddle-to-peak irradiance ratio is 0.8106 , while for a circular aperture is 0.7350 . In spite of that,this important physical error unfortunately propagates through many text books up to now.

\section{RESOLUTION AND SPECTRAL CONTENT}

Lord Rayleigh himself stated other factors to be taken into account when analysing the optical signals resolution. Among other factors, he pointed out the importance of color to study the diffraction gratings resolution and the use of color filters to that purpose. ${ }^{5} \mathrm{He}$ also discussed the influence of lenses and prisms aberrations on the resolution ${ }^{1}$ and also the quality of the optical surfaces ${ }^{5}$. In all these cases, such studies are related to light propagation through optical instruments in which the limiting pupils are constituted by the rectangular apertures of the dispersive elements: prisms and diffraction gratings. Besides, the light considered in these cases arises from sources of longitudinal geometries, such as the entrance slits of spectroscopes, spectrographs and monochromators. This study deals with the case of circular sources of small angular diameter observed through pupils which are also circular, such as those of the eyes and the photo and video cameras. It is true that the iris type mechanisms that determine the edges of pupils from polygonals do not produce in general a circular aperture. The effect of this difficulty was pointed out by Sommerfeld ${ }^{6}$, who explained the presence of pairs of light diffraction fans, as well as the disguising of other diffractive effects by them. Likewise, Gu and $\mathrm{Gan}^{7}$ recently studied the effect produced by the serrated or rugose edge of a circular pupil on the diffraction pattern. They found out that relations of the order of $5 \%$ between the rugosity and the radius of the pupil are enough to generate notable perturbations in the diffraction pattern.

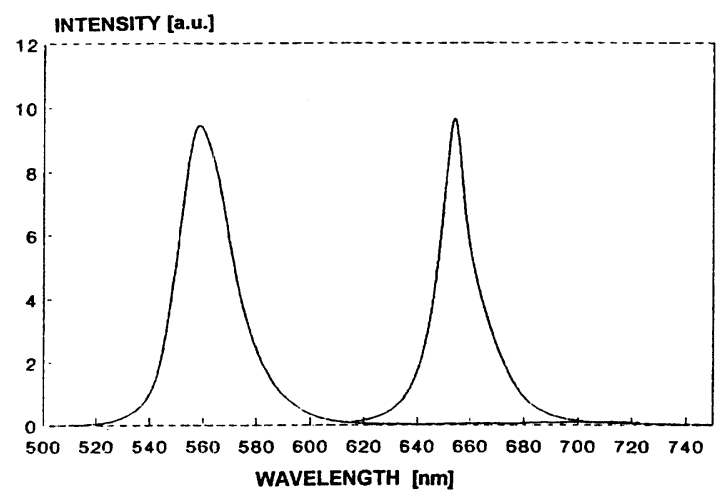

Fig. 1. Emission spectra of green (left) and red (right) LEDs. Their irradiances are normalized and expressed in arbitrary units. 
Light emitting diodes (LED) of $3 \mathrm{~mm}$ in diameter and in end-on observation were used in the experiments as light sources. Toshiba LEDs emitting in the green and red regions of the visible spectrum were used. These emissions are quasimonochromatic $(\Delta \lambda / \lambda<<1)$ and their spectra, observed in the laboratory, are shown in Figure 1. It is clearly noticed that the spectral content in the intermediate region corresponding to the yellow color is extremely low. The total irradiance for point light sources affected by diffraction through a circular aperture (Airy distribution) was calculated under Rayleigh conditions using the $560 \mathrm{~nm}$ (Green) and $650 \mathrm{~nm}$ (Red) wavelength values corresponding to the maxima of the LEDs spectra. The results are shown in Figure 2. It must be mentioned that the Rayleigh condition acquires now a degeneration as there are two ways of making the maxima and the first minima of the respective Airy distribution curves coincide, since this criterion is based on the coincidence of the null first derivatives. In Figure 2 -with the purpose of simplifying the representation and without implying too much loss of rigorousness- the graphics of the irradiance Airy patterns are represented without considering that the emissions are quasimonochromatic. A treatment dealing with such an aspect is being carried out following already known contributions ${ }^{8,9}$. Thus, as the angular radius of the Airy disk is
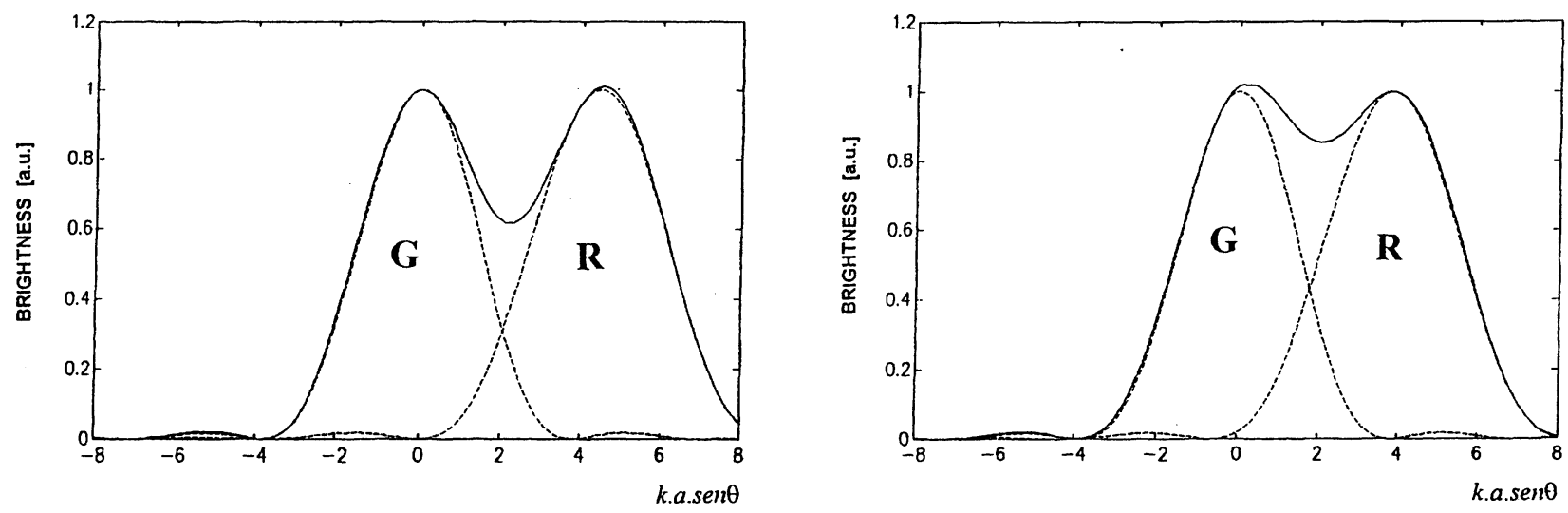

Fig. 2, a) Null first derivative condition of Rayleigh Criterion taking into account the central peak of the green Airy pattern and the first zero of the red Airy pattern. b) Null first derivative condition of Rayleigh Criterion taking into account the central peak of the red Airy pattern and the first zero of the green Airy pattern.

proportional to $\theta$, the red disk diameter will be larger than the green one. Then, if the green Airy pattern maximum and the red Airy pattern first minimum (Figure 2,a) overlap, the angular distance between the sources is equal to the angular radius $\theta$ of the red Airy disk. Likewise, the brightness corresponding to the minimum between both disks is equivalent to $\mathbf{0 , 6 1 4 4}$, i.e. it is lower than the value of 0,7350 corresponding to two point light sources of equal wavelength and brightness ${ }^{10}$. Its spatial resolution is thus secured. In the opposite case (Figure 2, b) the maximum of the red Airy pattern overlaps the minimum of the green Airy pattern, so the angular distance between the sources is less than $\theta$ and the brightness between them reaches a minimum of 0,8498 , being this value higher than the pertaining to two sources of equal wavelength and brightness. This implies, a priori, that the sources could not be distinguished at all. However, the easy visual observation and the photographic and videographic recordings of the non-spectral yellow false color produced by the overlapping of the red and green allow to secure the resolution.

\section{STUDY OF QUASIMONOCHROMATIC DISCOIDAL LIGHT SOURCES}

The possibility of raising the resolving power of optical instruments (eyes, photographic and television cameras, telescopes, microscopes and projectors) by the non-spectral yellow false color was confirmed when the red and green LEDs were observed with the naked eye as well as photographically. As the visual observation corresponds to the photographic one, only the latter results will be described. Both green and red LEDs were mounted on two orifices performed on a blackened metallic plate their centers separated $3 \mathrm{~mm}$. They were observed at distances of $2 \mathrm{~m}, 4 \mathrm{~m}$, and $8 \mathrm{~m}$ perpendicularly to the plate. The LEDs electric excitation was controlled by measuring their irradiance with a 1970 PR Spectra luminancemeter of the Pritchard type. An FG-20 Nikon camera with a Nikkor $f=135 \mathrm{~mm}$ teleobjective lens was 
used when photographing them. Kodak films of 400 ASA Elite (Color) and 100 ASA (Black and white) were utilized. With an exposition of tens of minutes, only the red or green LEDs were photographed, one by one or in pairs, at the different distances. With an exposition of fractions of seconds and using films for color slides, only the central peaks of the diffraction patterns, the dimensions of which sometimes correspond to the LEDs geometrical projection, were recorded. Once the red and green LEDs were photographed at the three distances, the films were developed in the laboratory. Image enlargements to $68 \mathrm{X}$ obtained by microscopy are shown in Figure 3, together with the dimensional description of the experiment. The enlargements were obtained using a PH Neofluor IM 35 Carl Zeiss microscope. Although the original images of the color slides are equal in size, the same does not occur with the microscopic images shown in Figure 3, owing to the spectral content of the microscope illumination lamp. In the upper part of Figure 3, the images of one and a pair of red LEDs (in black and white) can be compared. It is evident that at a distance of $8 \mathrm{~m}$, it cannot be distinguished whether it is a photograph of one or two LEDs. In the case of the red and green LEDs images, the non-spectral yellow false color allows the two sources to be easily distinguished. The electronically processed images obtained from the microscopic results of the color slides of a green and a red LED photograph are shown in the lower part of Figure 3. \{The impossibility of reproducing colors in this paper reduces the direct observation of beautiful original color images in Figures 3,4 and 5$\}$. The red and green LEDs and their images at a distance of $2 \mathrm{~m}, 4 \mathrm{~m}$ and $8 \mathrm{~m}$ enlarged by microscopy are represented in light and dark grey respectively on a black background. The non-spectral yellow false color represented in white becomes visible at four meters.

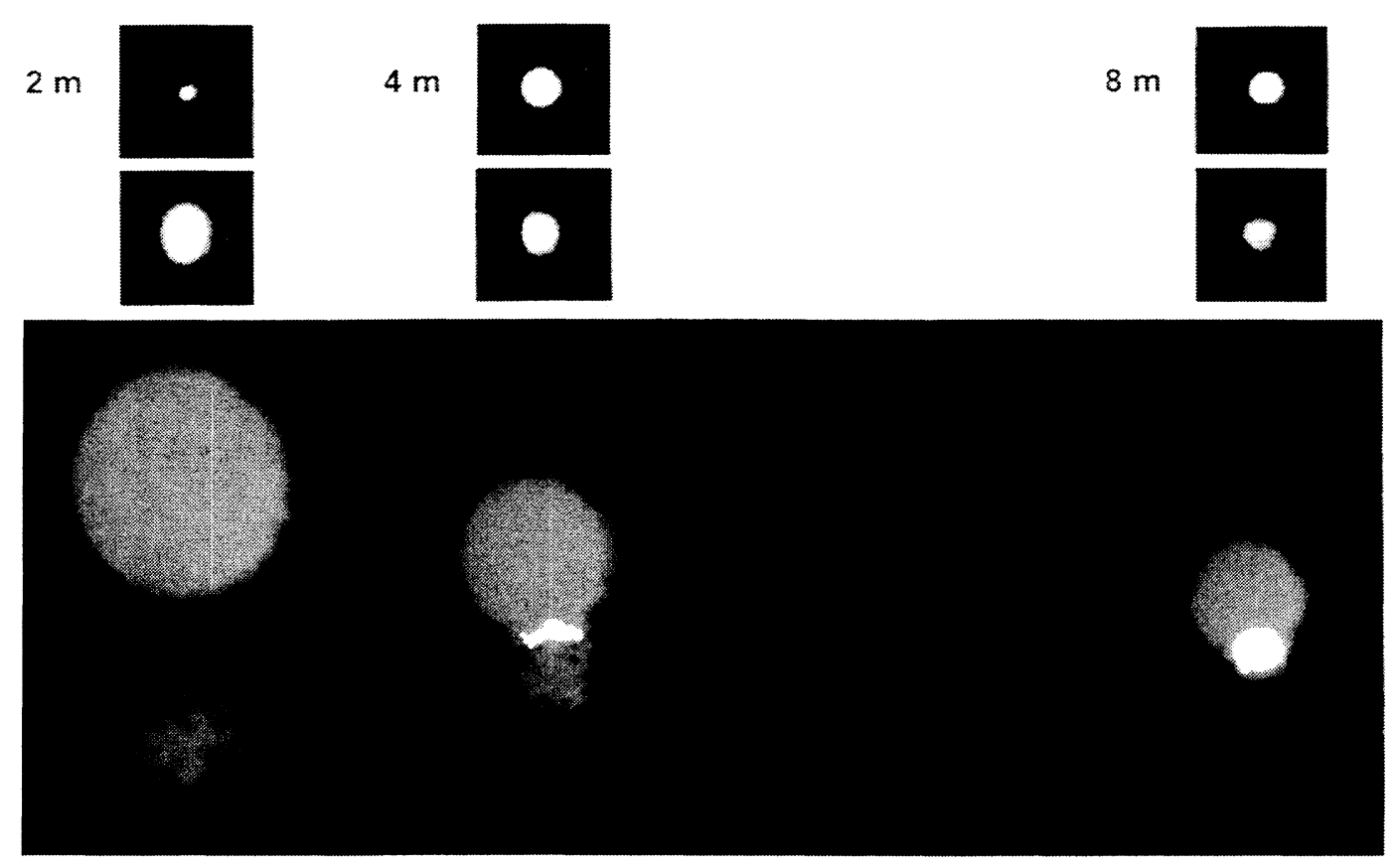

Figure 3

Fig. 3. In the upper part, the images of one and a pair of red LEDs can be compared at 2 $\mathrm{m}, 4 \mathrm{~m}$ and $8 \mathrm{~m}$. It is evident that at a distance of $8 \mathrm{~m}$, it cannot be distinguished whether it is a photograph of one or two LEDs. In the case of the red and green LEDs images, the non-spectral yellow false color allows the two sources to be easily distinguished. The electronically processed images obtained from the microscopic results of the color slides of a green and a red LED photograph are shown in the lower part. The red and green LEDs and their images at a distance of $2 \mathrm{~m}, 4 \mathrm{~m}$ and $8 \mathrm{~m}$ enlarged by microscopy are represented in light and dark grey respectively on a black background. The non-spectral yellow false color represented in white becomes visible at four meters. 
Likewise, the slides obtained in the experiment were observed microscopically introducing an incorrect focusing in the operation. The original image used is the one taken at a distance of $2 \mathrm{~m}$ as shown in Figure 3 . A sequence of four photographs at $68 \mathrm{X}$, the first one in focus and the others progressively out of focus, is shown in Figure 4 . In this Figure 4 as in Figure 3, the original color images were reproduced by means of an electronic process. The red and green LEDs are perfectly separated in the in focus microscope image, while in the out of focus images, the non-spectral yellow false color allows distinguishing the two light sources.

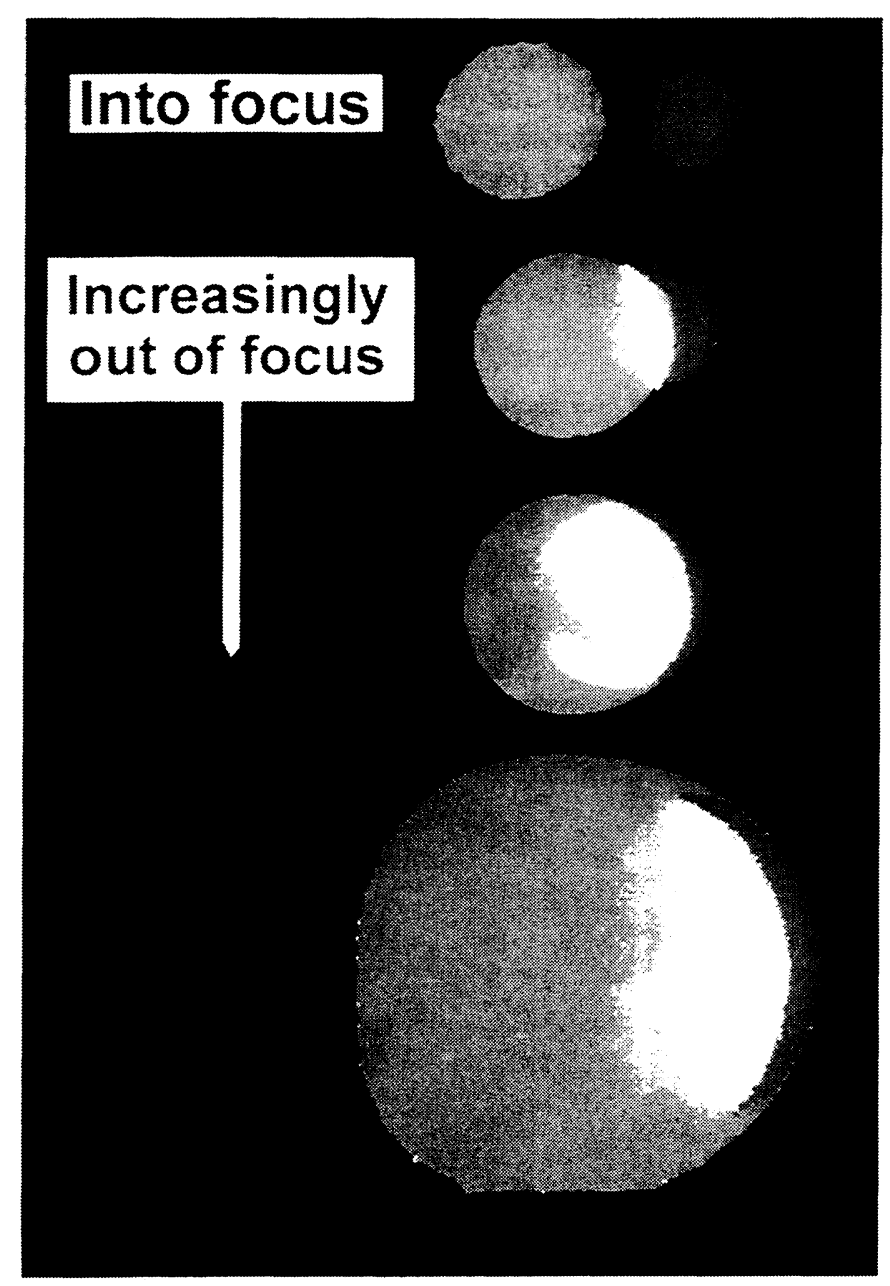

Figure 4

Fig. 4. Slides obtained in the experiment were observed microscopically introducing an incorrect focusing in the operation. The original image used corresponds to the one taken at a distance of $2 \mathrm{~m}$ as shown in Fig. 3. A sequence of four photographs at $68 \mathrm{X}$, the first one in focus and the others progressively out of focus, is shown. As in Fig. 3, the original color images were reproduced in a grey scale by means of an electronic process. The red and green LEDs are perfectly separated in the in focus microscope image, while in the out of focus images, the non-spectral yellow false color allows distinguishing the two light sources. 


\section{STUDY OF MONOCHROMATIC LASER LIGHT SOURCES}

The study of the possibility of raising the resolving power of optical instruments (eyes, photographic and television cameras, telescopes, microscopes and projectors) by the observation of the non-spectral yellow false color using red and green LEDs was extended to the observation of monochromatic light sources. Red and green He-Ne lasers were used in the experiment. The direct laser beams were combined to illuminate a pinhole $50 \mu \mathrm{m}$ in diameter in a coaxial fashion. When the overlapped Airy patterns are observed they look as an single Airy pattern that contains a non-spectral yellow false color. If the laser light emerging from the pinhole is analysed with a spectral apparatus, the images of its original spectral components are observed. A photographic reproduction of the spectral analysis of the non-spectral false color signal is shown in Figure 5. The non-spectral yellow false color Airy pattern is observed in the diffraction zero order. This Airy diffraction pattern seems to be produced by a circular pinhole pupil illuminated by yellow light. However, in the first order of diffraction, the Airy patterns of each of the spectral components -red and green- are clearly resolved. The experimental set-up is described in Figure 6.

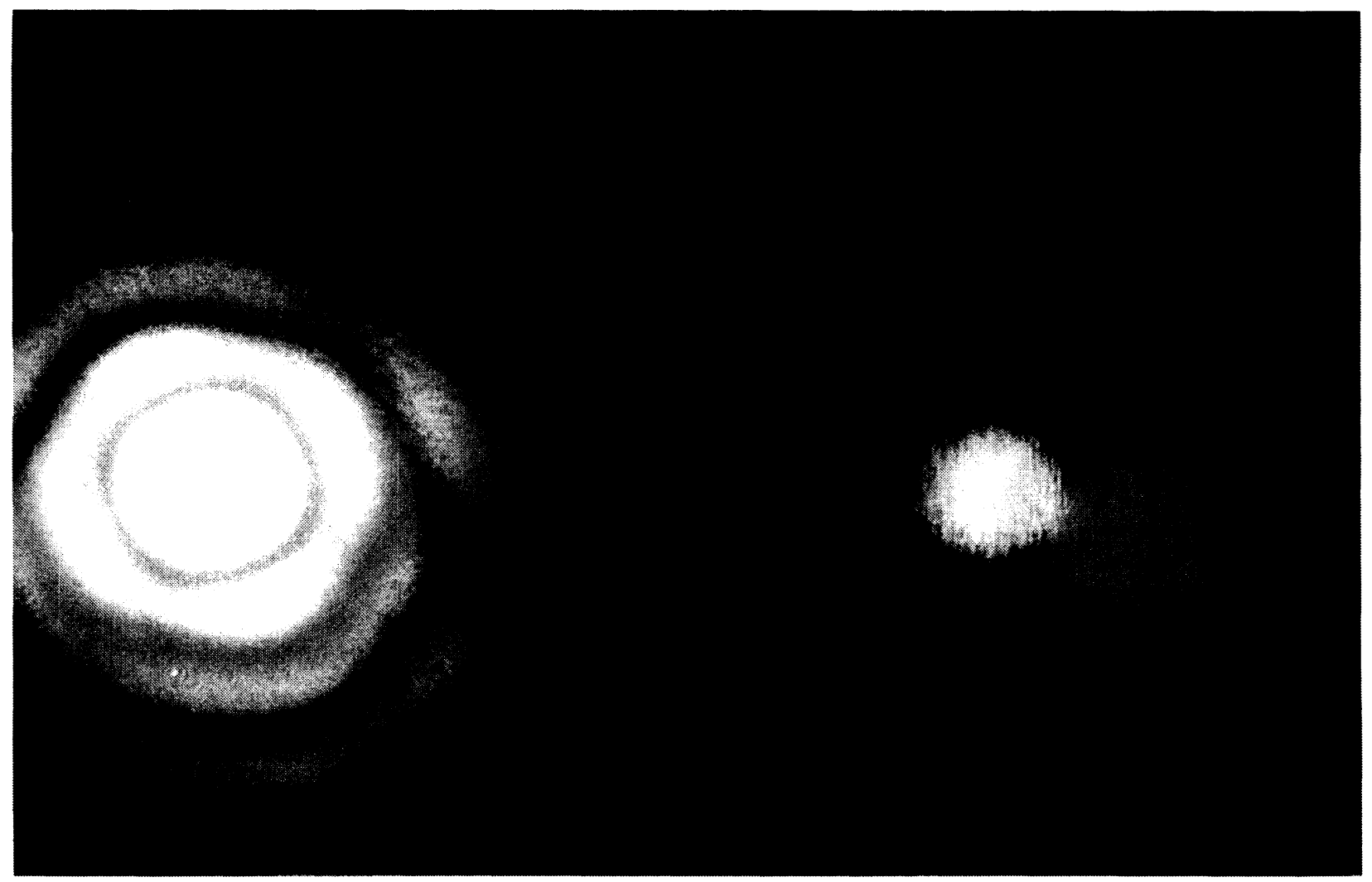

Figure 5

Fig. 5. The non-spectral yellow false color Airy pattern is observed in the diffraction zero order (Left). This Airy diffraction pattern seems to be produced by a circular pupil illuminated by yellow light. However, in the diffraction first order (Right), the Airy patterns of each of the spectral components -red and green- are clearly resolved. As in Fig. 3, the original color images were reproduced in a grey scale by means of an electronic process.

\section{CONCLUSIONS, PROJECTS AND PROPOSAL}

The non-spectral false color observation makes it possible to resolve light sources beyond the limit of resolution established by Rayleigh Criterion, i.e. the super-resolution observation is achieved. Experiments can be performed to allow the visualization of multijunction LEDs and a collection of bounded LEDs of different color emissions; the observation of 
biological specimens stained by many fluorocromes and of electrophoretogram with the overlapping of different color bands; the detection of images of stars with different spectral distributions and the recording of atmospheric disturbances due to the overlapping of light from sources of different spectral content can be mentioned among other cases.

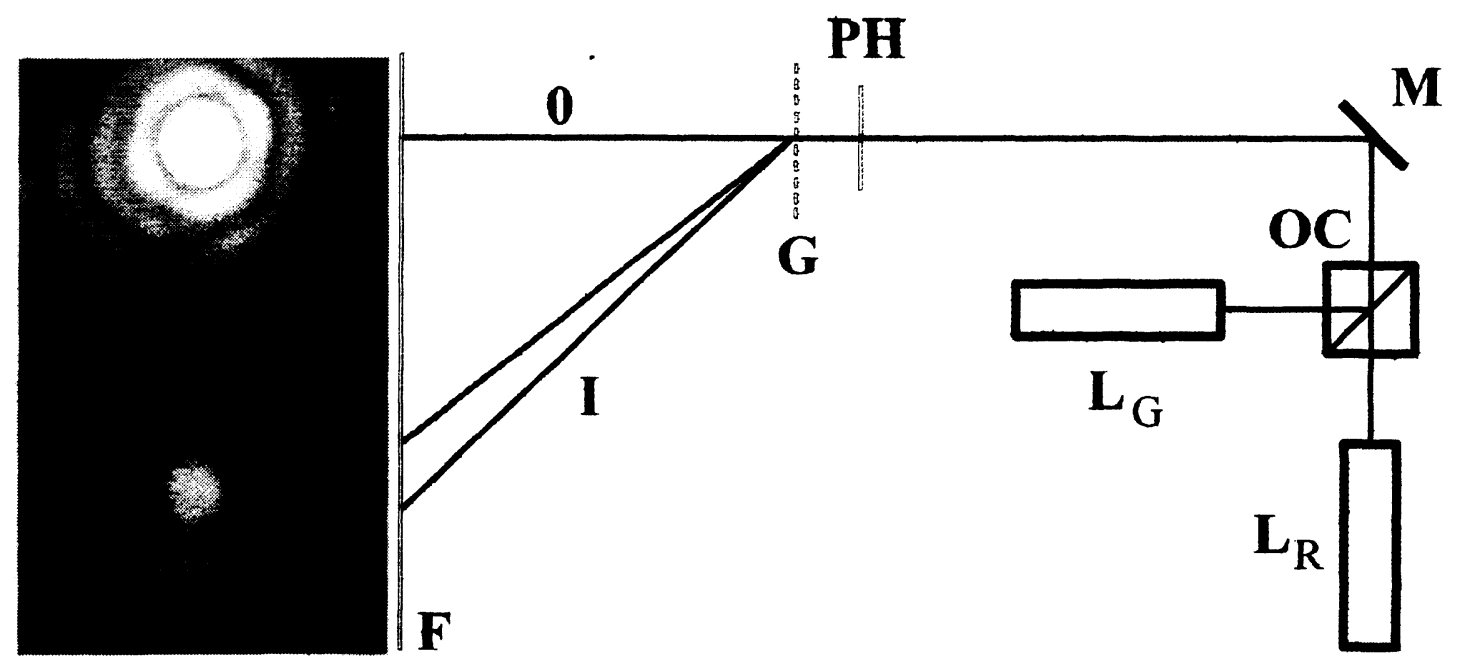

Fig. 6. Experimental set-up to photograph both diffraction orders observed at Fig. $5 . \mathrm{L}_{G}$ and $\mathrm{L}_{R}$ are He-Ne lasers emitting at green and red wavelengt; $\mathrm{OC}$ is an optical combiner of laser beams; $\mathrm{M}$ is a flat mirror; $\mathrm{PH}$ is a $\mathbf{5 0} \mu \mathrm{m}$ diameter pinhole to produce Airy diffraction patterns at both laser radiations; $G$ is a transmission diffraction grating and $F$ is the color film to record the zero and first diffracted orders.

It should be necessary to study the photographic and videographic materials used in the experimental observations and the processing of them, as well as the reproduction systems. Experience has demonstrated that small variation in the original characteristics of materials or processes produces considerable variation in the non-spectral false color, disguising the real relative contribution of the original spectral colors. Thus, the psychophysical analysis of the non-spectral false color is still useful to recognize such contributions. An observer without training is able to easily distinguish up to seven non-spectral false colors between red and green, while one with training is able to distinguish up to thirteen colors.

\section{ACKNOWLEDGMENTS}

The authors are very grateful to Dr. Ricardo Duchowicz for the LEDs spectra of Figure 1, to Eng. Pablo Ixtaina for the Pritchard luminancemeter measurings, to Dr. Néstor Carri for his help as regards microscopy, and to Lic. Enrique Campitelli for his suggestions. The authors are thankful for the partial financial support from Consejo Nacional de Investigaciones Científicas y Técnicas and Universidad Nacional de La Plata. CIOp depends on Consejo Nacional de Investigaciones Científicas y Técnicas and Comisión de Investigaciones Científicas de la Provincia de Buenos Aires, Argentina.

\section{REFERENCES}

1. John William Strutt (III Lord Rayleigh), "Investigations in Optics, with special reference to the spectroscope". Philosophical Magazine, Vol. VIII, pp. 261-274; pp. 403-411; pp. 477-486, 1879 and Vol. IX, pp. 40-55, 1880.

2. G. Bruhat, Optique, Masson et Cie., Editeurs, Paris, (6th. edition) pp. 247-248, 1965.

3. R. W. Ditchburn, Light, Blakie \& Son Limited, London, (2nd. edition) pp. 277-278, 1963

4. M. Born and E. Wolf, Principles of Optics, Pergamon Press, Oxford, (5th edition), p. 415, 1975.

5. John William Strutt (III Lord Rayleigh), "On the resolving power of telescopes", Philosophical Magazine, Vol. X, pp. $116-119,1880$ 
6. A. Sommerfeld, Optics. Lectures on Theoretical Physics, Vol. IV, Academic Press Inc., Publishers, New York, , pp. 233-235, 1954.

7. M. M. Gu and X. S. Gan, "Fresnel diffraction by circular and serrated apertures illuminated with an ultrashort pulsed-laser beam", Journal of the Optical Society of America, A, 13, 771- 778 (1996).

8. J. Bescós and J. Santamaría, "Formation of color images. Optical transfer functions for the tristimulus values", Photographic Science and Engineering, 21, 355-362 (1977).

9. H. S. Dhadwal and J. Hantgan, "Generalized point spread function for a diffraction-limited aberration-free imaging system under polychromatic illumination", Optical Engineering, 28, 1237-1240 (1989).

10. M. V. Ceressetto, T. S. Grigera, B. O'Donell, J. Sandoval y M. Garavaglia, "Sobre el criterio de resolución de Rayleigh para fuentes policromáticas" (In Spanish), Anales of the Asociación Física Argentina, Vol. 3, pp.192-195 (1991). 